\title{
Observations of Short-term Dips in the Orion Variables DD Serpentis and AB Aurigae
}

\author{
I.A. Verlyuk \\ Main Astronomical Observatory, National Academy of Sciences of Ukraine, \\ $252650 \mathrm{Kiev}$, Ukraine
}

\begin{abstract}
Short-term fadings (dips) in the range of a few seconds with amplitudes from $0^{\mathrm{m}} \cdot 3$ to 1.2 were discovered in the two $T$ Orions type variables DD Ser and AB Aur. Similar features were observed in T Tau and $\mathrm{R} \mathrm{Cr} B$ by other observers.
\end{abstract}

\section{Introduction}

The Orion variables (INA) of spectral types B-A are characterized by occasional sudden Algol-like fadings with amplitudes of about $0.5-1{ }^{\mathrm{m}} .0$ in the range from a few hours to several days. This kind of activity may by interpreted in terms of episodic condensation of grains in a stellar wind. Our observations reveal that similar features take place on time scales a short as several seconds.

\section{Observations}

The observations were obtained at the $60 \mathrm{~cm}$ telescope at the Mount Terskol Observing Station in North Caucasus (3100 m above sea level). They were obtained with a high-speed two-channel photometer (Zhilyaev et al. 1992). Simultaneous measurements of a reference star was performed to accurately determine atmospheric conditions during the time of observations. High time resolution monitoring of $\mathrm{AB}$ Aur and DD Ser was made in the $U B V$ bands in autumn of 1992 and 1993. Integration times in the range 0.1 to $0.5 \mathrm{sec}$ were used.

\section{Results}

In DD Ser, few dips were detected with amplitudes and durations in the range from $0^{\mathrm{m}} 3$ to $0^{\mathrm{m}} .7$ and $4-5 \mathrm{sec}$, respectively. One short-lived dip was detected in $A B$ Aur with an amplitude of the order of 1.2 . The duration of the latter was $1.7 \mathrm{sec}$. All dips show more or less symmetrical smooth light curves. Figs. 1-2 show that the reference star remained constant. Similar dips features were observed in T Tau and R CrB. As noted by Zajtseva (1980) the light curve of T Tau on 1977 October 30 , exhibits a few sudden fadings up to about $1^{\mathrm{m}}$ on 


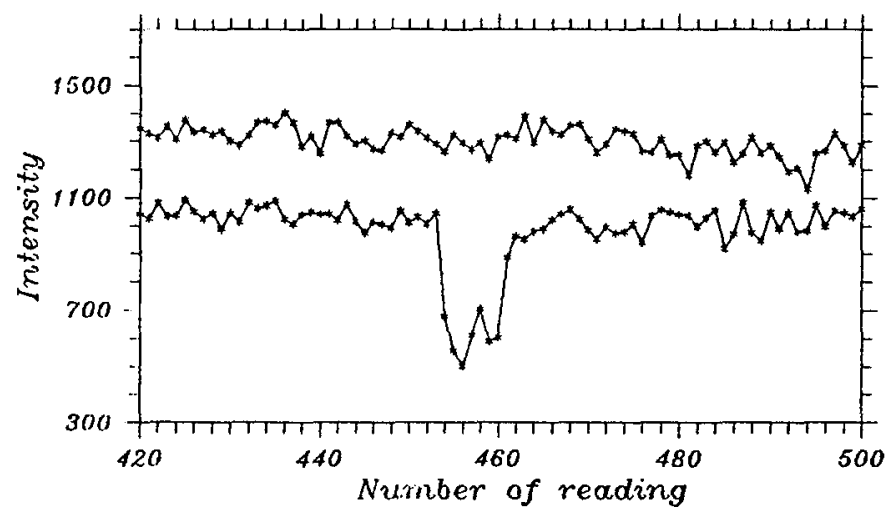

Fig. 1. DD Ser on 1992, July 6. B band. Time resolution: $0.5 \mathrm{sec}$. Top: reference star; bottom: variable.

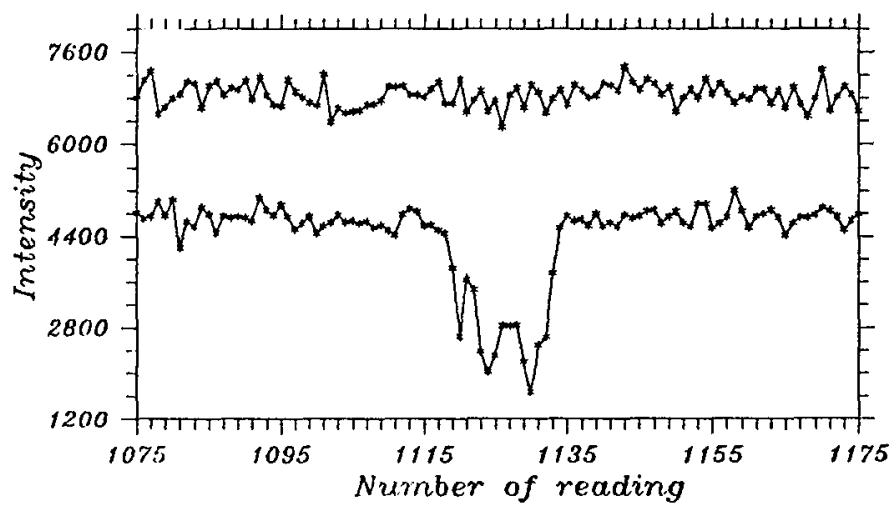

Fig. 2. AB Aur on 1992, August 21. Same parameters as Fig. 1

a time scale of $10 \mathrm{sec}$ in the $U$ band. Similar phenomena had been observed in $\mathrm{R} \mathrm{CrB}$ by Totochava (1977) during the deep minimum passage in 1976. One can imagine that dips observed in the cases of DD Ser and AB Aur are quite similar to those observed on $\mathrm{T}$ Tau and R CrB.

Acknowledgements: I wish to thank Dr. B.E. Zhilyaev for his kind assistance.

\section{References}

Totochava A.G., 1977, Photometric study of the RCB type stars, Ph.D. thesis, Odessa State University

Zajtseva G.V., 1980, in Flare Stars, Fuors and Herbig-Haro Objects, L. Mirzoyan (ed.), Yerevan, 1980, p. 61

Zhilyaev B.E., Romaniuk Ya.O., Svyatogorov O.A., 1992, AZh 69, 895 